\title{
Luminescence of singlet self-trapped excitons in $\mathrm{MgF}_{2}$
}

\author{
V N Kolobanov ${ }^{1}$, V V Mikhailin ${ }^{1}$, S P Chernov ${ }^{1}$, D A Spassky ${ }^{2}$, \\ V N Makhov ${ }^{3}$, M Kirm ${ }^{4}$, E Feldbach ${ }^{4}$ and S Vielhauer ${ }^{4}$ \\ ${ }^{1}$ Physics Department, M V Lomonosov Moscow State University, 119992 Moscow, Russia \\ ${ }^{2}$ Skobeltsyn Institute of Nuclear Physics, M V Lomonosov Moscow State University, \\ 119992 Moscow, Russia \\ ${ }^{3}$ P N Lebedev Physical Institute, 119991 Moscow, Russia \\ ${ }^{4}$ Institute of Physics, University of Tartu, 51014 Tartu, Estonia \\ E-mail: vkolobanov@yandex.ru
}

Received 23 June 2009, in final form 28 July 2009

Published 19 August 2009

Online at stacks.iop.org/JPhysCM/21/375501

\begin{abstract}
Fast $(\tau \sim 1.7 \mathrm{~ns})$ broadband (full width at half-maximum $=1.1 \mathrm{eV}$ ) vacuum ultraviolet $\left(\mathrm{VUV} ; h v=8.4 \mathrm{eV}\right.$ ) luminescence from $\mathrm{MgF}_{2}$ crystals has been detected at low temperature $(T<80 \mathrm{~K})$ and analyzed with techniques of cathodoluminescence and time-resolved VUV spectroscopy using synchrotron radiation. The VUV emission discovered has been attributed to the luminescence of spin-singlet self-trapped excitons (STEs). In contrast to the case for alkaline earth fluoride crystals with fluorite structure, the luminescence of the singlet STEs in $\mathrm{MgF}_{2}$ shows smaller Stokes shift than that of the triplet STEs, which is similar to 'typical' behavior observed for alkali halide crystals.
\end{abstract}

\section{Introduction}

The coexistence of different kinds of self-trapped excitons (STEs) is a characteristic feature of insulating crystals, and studies of the nature and the existence conditions for different STEs attract great attention from researchers. According to modern understanding, the STEs decay radiatively from one or more local minima on the adiabatic potential energy surfaces for the spin-singlet and spin-triplet states of the lowest orbital energy (see [1] and references therein). From the singlet states, fluorescence with short lifetime is emitted, whereas from the triplet states, luminescence with long lifetime is observed. As was clarified for alkali halide crystals [2] there exist three kinds of STE lattice configurations: type I corresponding to the 'on-center' or ' $\mathrm{V}_{\mathrm{k}}+\mathrm{e}^{-}$, configuration, and types II and III corresponding to two 'off-center' configurations with different values of the asymmetric lattice relaxation.

One of the main methods used in the studies of different kinds of STEs is time-resolved photoluminescence spectroscopy. It is well known that in alkaline earth fluoride crystals with fluorite structure $\left(\mathrm{CaF}_{2}\right.$ and $\left.\mathrm{SrF}_{2}\right)$, the triplet and singlet STEs coexist and their emission bands overlap spectrally [3, 4]. So, experimental identification of the fast (singlet, $\tau \approx 10 \mathrm{~ns}$ ) and slow (triplet, $\tau \approx 50 \mu \mathrm{s}$ ) components of the STE luminescence in these crystals is only possible with the technique of time-resolved spectroscopy [3-6].

The lattice configuration of STEs in fluorite crystals is very similar to a pair of adjacent $F$ and $H$ centers [7]. This means that the hole component of the STE- the $\mathrm{F}_{2}^{-}$molecular ion-occupies the single-anion site, being aligned along the $\langle 111\rangle$ direction, and its configuration is close to that of the $\mathrm{H}$ center, whereas the electron charge density of the STE is concentrated on the anion vacancy. Note that in contrast to the alkali halide case, the lattice configuration of STEs in fluorite crystals differs from that of $\mathrm{V}_{\mathrm{k}}$ centers-self-trapped holes (STHs) - not only by the translation of the ions but also in the orientation of the $\mathrm{F}_{2}^{-}$molecular ions, which are aligned along the $\langle 100\rangle$ direction in the $\mathrm{V}_{\mathrm{k}}$ centers. Such strong lattice relaxation with additional 'rotation' of the $\mathrm{F}_{2}^{-}$molecular ion explains the rather large Stokes shift for STEs in fluorite crystals (between 6 and $7 \mathrm{eV}$ ) and can be related to the offcenter geometry of both kinds of STEs.

For $\mathrm{MgF}_{2}$ having the rutile lattice structure, only a slow (triplet, $\tau \approx 6.4 \mathrm{~ms}$ ) component of the STE luminescence [8] has been revealed and studied so far. To the best of our knowledge, there have been no publications where emission from $\mathrm{MgF}_{2}$ crystals in the vacuum ultraviolet (VUV) spectral range has been considered. The absence of data on VUV 
luminescence spectroscopy for such a well-known compound as $\mathrm{MgF}_{2}$ is due to the experimental difficulties in carrying out studies in the VUV region, especially for luminescence with time resolution, where special optics and detectors should by applied. In the present paper we report on the discovery and spectroscopic studies of fast VUV luminescence from $\mathrm{MgF}_{2}$ using synchrotron radiation as an excitation light source as well as stationary electron beam excitation. This study should have significant impact on practical applications because $\mathrm{MgF}_{2}$ is a well-known UV/VUV optical material.

\section{Experimental details}

The measurements under VUV photon excitation were performed at the SUPERLUMI station [9] of HASYLAB at DESY, using synchrotron radiation from the DORIS storage ring (in the spectral range up to $\sim 20 \mathrm{eV}$ ). The time-resolved emission and excitation spectra of the VUV luminescence were recorded with a Pouey-type monochromator (typical spectral resolution $\Delta \lambda=20 \AA$ ) equipped with an R6838 (Hamamatsu) solar-blind photomultiplier tube (PMT). The timing properties of this PMT were quite poor for the measurements of luminescence decay curves in the range around $1 \mathrm{~ns}$, but suit well the conditions for the measurements of time-resolved spectra. Discrimination between the fast and slow emission in the spectra was achieved by measuring signals within 'short' and 'long' time windows $(\Delta t)$ delayed (by $\delta t$ ) with respect to the excitation pulses of synchrotron radiation. For the measurements of VUV luminescence decay curves a CsI sensitized microsphere plate detector was used which has rather poor sensitivity in the spectral range of interest but provides a good time resolution of $\sim 0.1 \mathrm{~ns}$. A 0.3 m Czerny-Turner-type monochromator-spectrograph SpectraPro-308i (Acton Research Inc.) with an R6358P (Hamamatsu) PMT was applied for measuring excitation and emission spectra of UV/visible luminescence. Measured emission spectra were not corrected for the spectral response of the detection system.

Some measurements were performed also at the BW3 undulator beamline of HASYLAB at DESY under excitation by XUV photons (100-600 eV). In these measurements a high throughput $0.4 \mathrm{~m}$ VUV monochromator was used together with a microchannel plate-type PMT (Hamamatsu 1645 U-09) providing the spectral working range $\lambda=110-500 \mathrm{~nm}$ at a spectral resolution of $\sim 6 \AA$ [10]. The effective time resolution of the detection system was 300 ps (FWHM), with a 130 ps length of the exciting synchrotron radiation pulses.

Cathodoluminescence spectra and thermoluminescence glow curves after electron beam irradiation were recorded in the temperature range between 5 and $300 \mathrm{~K}$. In these measurements the luminescence was detected by two spectrometers working in different spectral ranges: by a double-grating VUV spectrometer equipped with a solar-blind PMT (Hamamatsu R6838) and by the spectrometer for the UV/visible spectral range equipped with a Hamamatsu photon counting head H6240 [11]. The characteristics of the electron gun were as follows: tunable electron energy in the range 1$30 \mathrm{keV}$, electron current $10-100 \mathrm{nA}$, a typical spot size of $\sim 0.5 \mathrm{~mm}^{2}$.

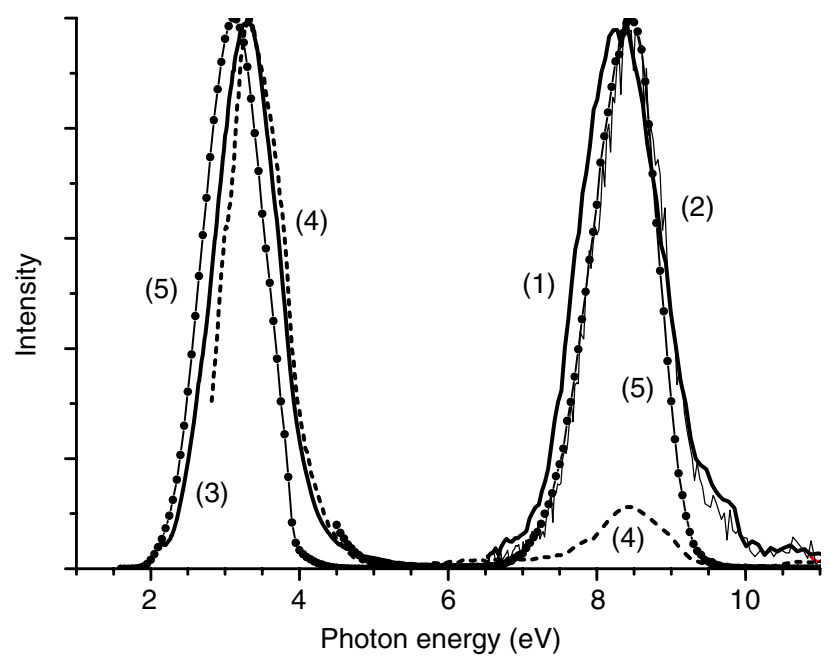

Figure 1. Normalized emission spectra of $\mathrm{MgF}_{2}$ crystals under excitation with VUV photons: (1) $h v=13.2 \mathrm{eV}, T=9 \mathrm{~K}$; (2) $h v=13.5 \mathrm{eV}, T=9 \mathrm{~K}$; another sample; (3) $h v=11.4 \mathrm{eV}$, $T=9 \mathrm{~K}$; or with XUV radiation (4): $h v=150 \mathrm{eV}, T=18 \mathrm{~K}$ and with an electron beam (5): $E_{\mathrm{e}}=5 \mathrm{keV}, I_{\mathrm{e}}=0.5 \mu \mathrm{A}, T=5.5 \mathrm{~K}$. In the case of excitation with VUV photons or an electron beam the spectra in VUV and UV/visible spectral ranges were measured with different spectrometers.

Several nominally pure single-crystalline samples of $\mathrm{MgF}_{2}$ of different origin were studied in this work. To ensure clean surfaces, the samples were cleaved prior to mounting onto the sample holder of a liquid helium cryostat. The orientation of the crystallographic axes of $\mathrm{MgF}_{2}$ samples was arbitrary with respect to the polarization vector of the synchrotron radiation.

\section{Results and discussion}

Figure 1 shows typical emission spectra of $\mathrm{MgF}_{2}$ single crystals excited by VUV or XUV radiation as well as by electrons with the energy of $5 \mathrm{keV}$. The shapes of the spectra are slightly different, especially in the UV/visible spectral range, depending on the origin of the sample and on the excitation type and energy. This can be explained by the presence of various defects and/or impurities in different samples and by variation of the penetration depth for different kinds of irradiation. It should also be noted that the spectra were measured with different spectrometers for different kinds of excitations. However, for all the samples there is a wellpronounced emission band in VUV region centered at $8.4 \mathrm{eV}$ with a FWHM of $\sim 1.1 \mathrm{eV}$ at $T \sim 9 \mathrm{~K}$. The decay time of this emission measured under both VUV and XUV excitation was found to be $\sim 1.7 \mathrm{~ns}$. The decay curve under XUV excitation is slightly non-exponential with a faster initial stage (figure 2), typical for such excitation (see, e.g., [12]). All crystals also show broadband slow emission with a maximum near $3.2 \mathrm{eV}$ with a similar FWHM of $\sim 1.0 \mathrm{eV}$, which corresponds to the luminescence of well-studied triplet STEs [8].

Excitation spectra of luminescence at 8.4 and $3.2 \mathrm{eV}$ are shown in figure 3 . The VUV luminescence at $8.4 \mathrm{eV}$ is efficiently excited under both direct optical creation of excitons 


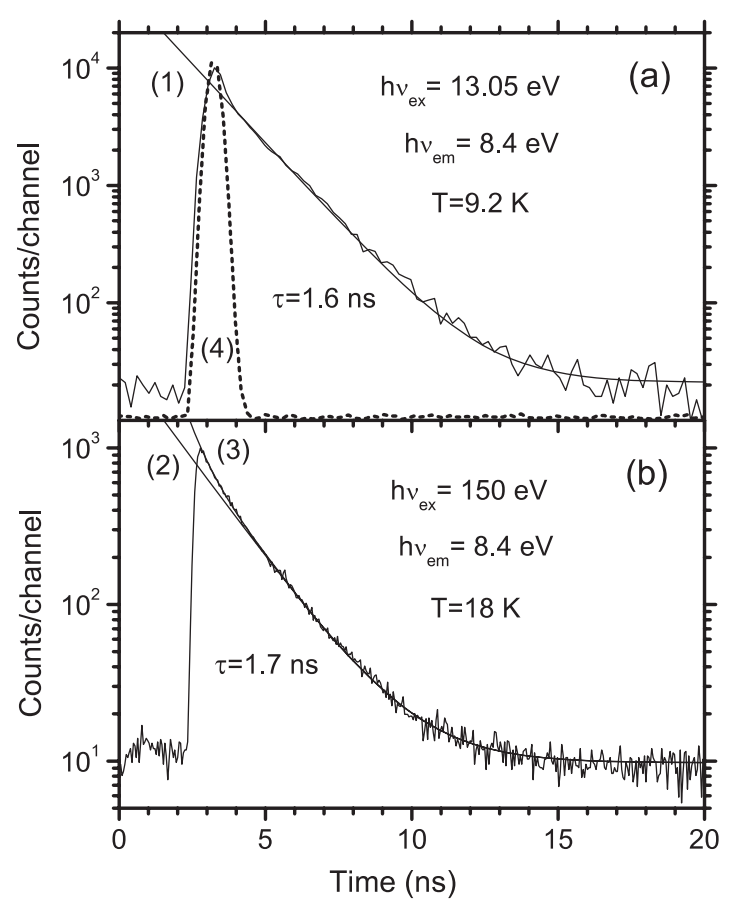

Figure 2. Decay curves of $8.4 \mathrm{eV}$ luminescence from $\mathrm{MgF}_{2}$ crystals excited with pulsed VUV ((a) $h v=13.05 \mathrm{eV}, T=9.2 \mathrm{~K}$ ) and XUV ((b) $h v=150 \mathrm{eV}, T=18 \mathrm{~K}$ ) synchrotron radiation. The curves were fitted with a single-exponential decay with $\tau=1.6 \mathrm{~ns}$ (1) for VUV excitation and with a single-exponential decay with $\tau=1.7 \mathrm{~ns}$ (2) or two-exponential decay with $\tau_{1}=1.7 \mathrm{~ns}$ and $\tau_{2}=0.5 \mathrm{~ns}$ (3) for XUV excitation. In the curve measured under VUV excitation the initial narrow peak is due to scattered light coming from the excitation pulse of the synchrotron radiation (4).

at $11.7 \mathrm{eV}<h v<12.4(12.6) \mathrm{eV}$ and as a result of electronhole recombination at $h v>12.4(12.6) \mathrm{eV}$ (the band-gap energy $E_{\mathrm{g}}$ of $\mathrm{MgF}_{2}$ is estimated in [13] as $12.4 \mathrm{eV}$ for the polarization vector of the incident light parallel to the optical axis of the crystal and $12.6 \mathrm{eV}$ for the polarization vector of the incident light perpendicular to the optical axis). The excitation onset of VUV luminescence is situated at $\sim 11.75 \mathrm{eV}$ and is shifted by $\sim 0.5 \mathrm{eV}$ to higher photon energies compared to the excitation onset for triplet STEs. In the initial parts of the spectra the observed modulations (minima) are related to the peaks in fundamental absorption spectrum of the crystal corresponding to excitonic absorption of highly polarized synchrotron radiation by anisotropic $\mathrm{MgF}_{2}$ crystal (installed without control of the orientation of its crystallographic axes with respect to the exciting light polarization). In particular, the excitation spectra for both emissions show a deep minimum at $\sim 12.3 \mathrm{eV}$, corresponding well to the maximum in the low temperature reflection spectrum of $\mathrm{MgF}_{2}$ measured for the same crystal simultaneously with the excitation spectra (see figure 3).

The temperature dependences of the luminescence intensities at 3.2 and $8.4 \mathrm{eV}$ under electron excitation are shown in figure 4 . The visible non-zero intensity of the emission at $3.2 \mathrm{eV}$ in the temperature range $100-200 \mathrm{~K}$ is due to the presence of some other (extrinsic) emissions, which was proved by careful measurements of emission spectra at

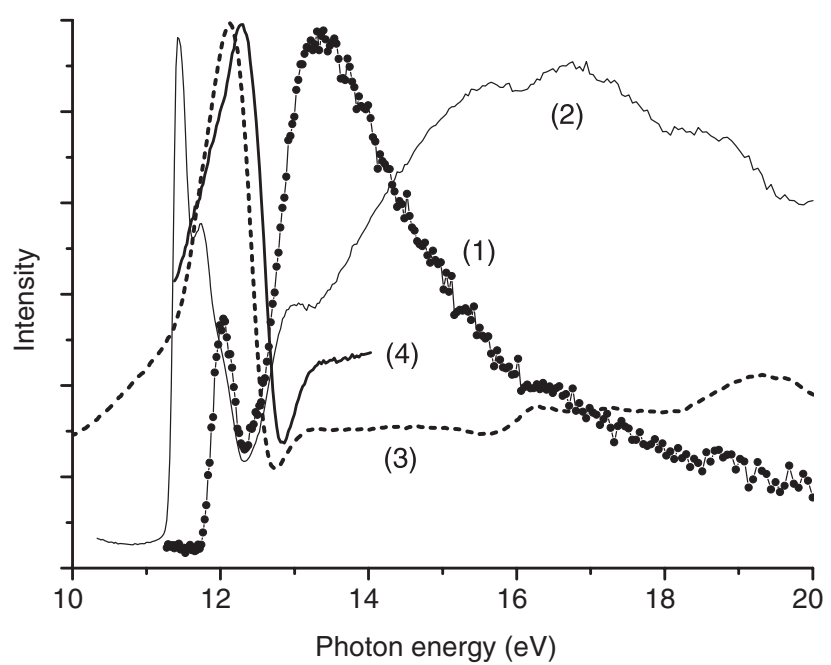

Figure 3. Excitation spectra of $8.4 \mathrm{eV}$ (1) and $3.2 \mathrm{eV}$ (2) luminescence from $\mathrm{MgF}_{2}$ at $9 \mathrm{~K}$. Reflection spectra of $\mathrm{MgF}_{2}$ at room temperature (3) and $9 \mathrm{~K}(4)$.

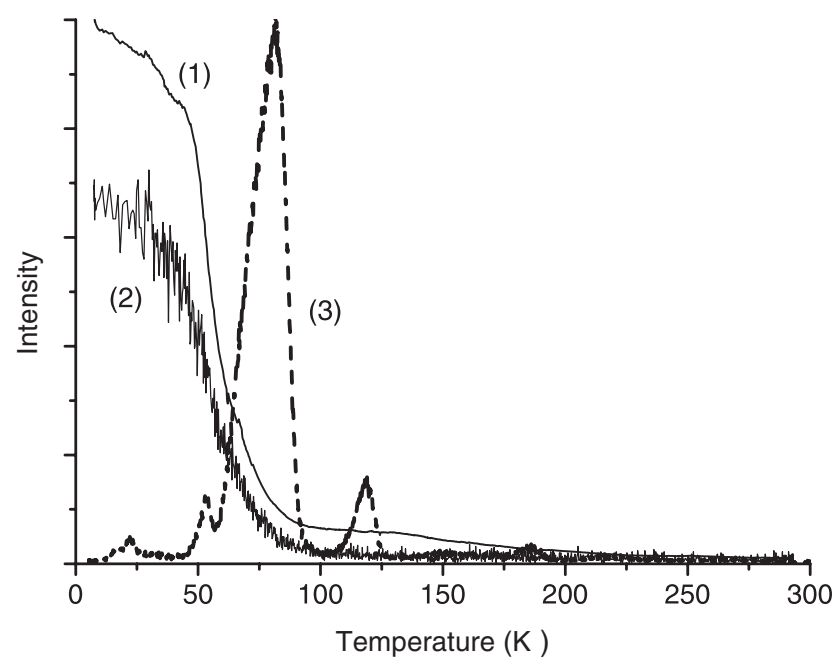

Figure 4. Temperature dependences of luminescence intensities for $\mathrm{MgF}_{2}$ emissions at 3.2 (1) and 8.4 (2) eV under electron $\left(E_{\mathrm{e}}=5 \mathrm{keV}, I_{\mathrm{e}}=0.5 \mu \mathrm{A}\right)$ excitation. Thermoluminescence glow curve (3) recorded from the $\mathrm{MgF}_{2}$ crystal at a heating rate of $10 \mathrm{~K} \mathrm{~min}^{-1}$ after irradiation with an electron beam at $5.5 \mathrm{~K}$. The photon energy of the analyzing monochromator was set at $3.2 \mathrm{eV}$.

different temperatures. As can be seen in the figure, the VUV luminescence of $\mathrm{MgF}_{2}$ crystals is thermally quenched (the intensity is decreased by $50 \%$ ) at $T>55 \mathrm{~K}$ and the temperature dependences of the 3.2 and $8.4 \mathrm{eV}$ emissions practically coincide. The latter property indicates that the natures of the thermal quenchings for the two kinds of emissions should be the same. By fitting this dependence with the standard Mott formula, the activation barrier for thermal quenching can be estimated at the value of $\sim 30 \mathrm{meV}$. The measurements of the thermoluminescence glow curve after irradiation with $5 \mathrm{keV}$ electrons have shown that the main peak of the glow curve at $82 \mathrm{~K}$ is observed just in the same temperature interval where thermal quenching of both kinds of emissions takes place (see figure 4). The glow curve 
was recorded for the emission at $3.2 \mathrm{eV}$ corresponding to the luminescence of triplet STEs, but actually the emission spectrum of the thermoluminescence was not analyzed in detail, leaving space for possible emission from centers other than triplet STEs. Special attention was paid to the VUV region, but thermally stimulated emission of $\mathrm{MgF}_{2}$ in the VUV was not detected.

The combination of the observed properties of VUV luminescence from $\mathrm{MgF}_{2}$ crystals, namely: (i) the energy position of the excitation onset in the region of direct optical creation of free excitons (at slightly higher energy than that for triplet STEs), (ii) fast (nanosecond) decay corresponding to allowed transitions and (iii) thermal quenching at $T>55 \mathrm{~K}$, allows us to ascribe this emission to luminescence of singlet STEs. As was already mentioned, for alkaline earth fluoride crystals with fluorite structure the triplet and singlet STE emission bands nearly coincide, the Stokes shift (with respect to the energy of free exciton creation) being slightly larger for singlet STEs than for triplet STEs [3-6]. On the other hand, luminescence spectra of different kinds of STEs in alkali halide and silver halide crystals are spectrally resolved (see [1] and references therein) and the Stokes shift of emission is smaller for singlet-type STEs than for triplet-type STEs corresponding to the on-center and off-center spatial configurations of STEs respectively.

For fluorite crystals the slightly larger Stokes shift for singlet STEs than for triplet STEs suggests even larger lattice relaxation for the former kinds of STEs. This is not the case for $\mathrm{MgF}_{2}$ with different crystal structure of a rutile type. In $\mathrm{MgF}_{2}$ the STHs were identified in [14], where it was shown that $V_{k}$ centers in $\mathrm{MgF}_{2}$ have the two-center structure of nearly $\langle 011\rangle$ oriented $\mathrm{F}_{2}^{-}$molecular ions sharing the hole charge equally. The results of our measurements under photon and electron excitation clearly show that both kinds of STEs in $\mathrm{MgF}_{2}$ can be created as a result of recombination of electrons with STHs. However, like for the case of fluorite crystals, the lattice configuration of triplet STEs in $\mathrm{MgF}_{2}$ is not of the $\left(\mathrm{V}_{\mathrm{k}}+\mathrm{e}^{-}\right)$ type but is close to a pair of adjacent $F$ and $H$ centers [15] requiring a rather strong lattice relaxation (the orientation of the $\mathrm{F}_{2}^{-}$molecular ion in the $\mathrm{H}$ center does not correspond to the direction of any F-F bond in the lattice [14]). The respective Stokes shift is really large: $\sim 8.5 \mathrm{eV}$. The relatively small Stokes shift for the luminescence band of singlet STEs $(\leqslant 3.5 \mathrm{eV})$, i.e. a relatively small lattice relaxation for singlet STEs, indicates that STEs of this type in $\mathrm{MgF}_{2}$ can have nearly on-center lattice configurations of the $\left(\mathrm{V}_{\mathrm{k}}+\mathrm{e}^{-}\right)$type.

For alkali halide crystals the lattice configuration of STE, i.e. the degree of off-center relaxation, correlates with the socalled Rabin-Klick parameter $S / D$ [2], where $S$ is defined as the separation of two adjacent halide ions (along a $\langle 110\rangle$ axis) minus twice the halide ion radius, $D$ is the diameter of the halogen atom. The ratio $S / D$ is in fact the measure of space available for STEs to show off-center relaxation. For small values of the parameter $S / D$ only on-center kinds of STEs are observed, whereas for large $S / D$ only off-center STEs are created. For intermediate $S / D$ values the two kinds of STEs coexist in the crystals (in some cases together with one more STE type having an intermediate value of the lattice relaxation). The direct application of the above parameter for $\mathrm{MgF}_{2}$ and fluorite crystals is not justified since in these crystals the lattice relaxation during exciton self-trapping involves the rotation of the $\mathrm{F}_{2}^{-}$molecular ion in addition to translational movement. In crystals with fluorite structure there exist interstitial (hollow) sites in each cube of fluoride ions having no metal ion at its center. Thus, qualitatively we can note that in fluorite crystals there is a lot of free space available for off-center STE relaxation with the alignment of the $\mathrm{F}_{2}^{-}$molecular ion along the $\langle 111\rangle$ axis. In $\mathrm{MgF}_{2}$, the rutile structure having much denser packing is not so favorable for the off-center lattice relaxation of STEs as in fluorite crystals and the two kinds of STEs coexist: singlet STEs possess stable on-center lattice configurations whereas such lattice configurations are still unstable for triplet STEs, which relax to off-center configurations.

Similar to results obtained for fluorite crystals $[5,6]$ as well as for alkali halides [16, 17], the excitation onset of singlet STE luminescence in $\mathrm{MgF}_{2}$ is shifted to higher photon energies compared to that of triplet STE luminescence (situated at $\sim 11.75$ and $\sim 11.25 \mathrm{eV}$, respectively). In fact, due to the presence of secondary-order radiation in the spectrum of exciting synchrotron radiation, which cannot be removed because of the absence of suitable optical filters in this VUV region, it cannot be experimentally excluded that the excitation of singlet STE luminescence may occur at photon energies below the onset at $11.75 \mathrm{eV}$; however, its excitation efficiency in this region is very low, in any case. In previous studies the excitation onset of singlet STE luminescence was usually attributed to the creation of higher energy $(n \geqslant 2)$ free excitons. According to [17] the lowest energy $(n=1)$ free exciton in alkali halides relaxes more preferentially into the off-center STE configuration because of the small radius of the $n=1$ exciton, which leads to strong off-center instability. In the case of $\mathrm{MgF}_{2}$, similar to that for fluorite crystals [5, 6], the excitation onsets for triplet and singlet STEs are both situated at photon energies below the main excitonic peak in the reflection spectrum, i.e. the offsets cannot be definitely attributed to the creation of $n=1$ and $n \geqslant 2$ free excitons, respectively. It is very probable that in the regions very near the offsets the extrinsic luminescence (luminescence of near-impurity or near-defect STEs) can give a remarkable contribution to the observed spectra.

One more feature of the excitation spectra which should be mentioned is that the shape of the spectra for triplet and singlet STE luminescence is strongly different in the region of band-to-band transitions. For singlet STEs the efficiency of luminescence excitation after reaching the maximum just above the band-gap decreases continuously with the excitation energy. This means that the efficiency of the singlet STE creation as a result of electron-hole recombination depends on the kinetic energy of the hot electrons and holes created, i.e. on the electron-hole separation distance. Contrary to the singlet STE case, the efficiency of luminescence excitation for the triplet STE reaches its maximum at photon energies exceeding the band-gap by several eV. Although the excitation spectrum of the triplet STE luminescence shows typical modulations related to variation with energy of the absorption and reflection 
coefficients, the common trend of the spectrum behavior can be considered as being anti-correlated with the spectrum for singlet STEs. Such behavior could be the result of the competition of the creation of singlet and triplet STEs, which depends on the kinetic energy of the electrons and holes created.

The presence of a very deep minimum in the excitation spectra for both kinds of STEs at $\sim 12.3 \mathrm{eV}$ cannot be explained by just a large reflection coefficient of $\mathrm{MgF}_{2}$ for this peak, and so the near-surface losses should be taken into account (see, e.g., [6]). The energy position of this minimum corresponds to maximum efficiency of direct optical creation of excitons, which means that free excitons in $\mathrm{MgF}_{2}$ can migrate for rather long distances before self-trapping. On the other hand, the modulations of the excitation spectra in the region of band-to-band transitions are rather weak, i.e. the near-surface losses due to migration of free charge carriers as well as due to possible migration of STEs or STHs are quite small. Unfortunately, because of the absence of absolute reflection coefficients of low temperature reflection for $\mathrm{MgF}_{2}$ we were not able to perform simulations of excitation spectra according to the diffusion model (using Kramers-Krönig relations for the absorption spectrum calculation) similar to those performed in [6] for other fluorite crystals. It should be again noted that the orientation of the anisotropic $\mathrm{MgF}_{2}$ crystal studied with respect to the exciting light polarization was not controlled in the experiments performed, which makes the respective analysis even more complicated. However, we can note that the dip at about $11.6 \mathrm{eV}$ in the excitation spectrum of triplet STE emission correlates well with the maximum in the absorption spectrum of $\mathrm{MgF}_{2}$ for exciting light polarization parallel to the $c$-axis of the crystal, whereas the deep minimum at $\sim 12.3 \mathrm{eV}$ correlates with maximum in the absorption spectrum of $\mathrm{MgF}_{2}$ for exciting light polarization perpendicular to the $c$-axis of the crystal [13].

The nature of the thermal quenching of the STE luminescence in $\mathrm{MgF}_{2}$ is still under discussion. One could try to take into account the correspondence of the main thermoluminescence peak and the temperature range of the STE luminescence quenching. This peak definitely cannot be due to the release of electrons from some traps and subsequent recombination of electrons with STHs; otherwise VUV luminescence of singlet STEs would also be observed in thermoluminescence. The temperature range of thermal quenching corresponds well with thermally activated migration of $\mathrm{H}$ centers [18]. Such migration will result in recombination of $\mathrm{H}$ centers with distant $\mathrm{F}$ centers that can give rise to the appearance of STE luminescence (of a triplet type) observed in the dominant thermoluminescence peak at $82 \mathrm{~K}$. Thermally activated mobility of $\mathrm{H}$ centers could also be the main reason for thermal quenching for STE luminescence due to the thermal conversion of STEs having the off-center configuration (adjacent $\mathrm{F}-\mathrm{H}$ pairs) into more distant pairs of $\mathrm{H}$ and $\mathrm{F}$ centers instead of radiative recombination. However, for the on-center STEs such mechanism cannot explain the thermal quenching of the luminescence. Furthermore, as was shown in [8], the conversion of STEs in $\mathrm{MgF}_{2}$ into $\mathrm{F}-\mathrm{H}$ pairs at larger separation occurs at higher temperatures near $190 \mathrm{~K}$. It is very unlikely that thermal quenching of luminescence due to 'conventional' crossing of excited state potential curves with the ground state occurs at the same temperatures for both kinds of STEs. Thermally activated migration of STHs with subsequent recombination with electrons at some traps (before the creation of self-trapped excitons due to recombination of conduction electrons with STHs) could be the common process leading to thermal quenching of luminescence for both oncenter and off-center STEs.

\section{Conclusions}

Fast (nanosecond) broadband VUV luminescence peaked at $8.4 \mathrm{eV}$ has been detected from $\mathrm{MgF}_{2}$ crystals at low temperature $(T<80 \mathrm{~K})$ and analyzed with the technique of time-resolved VUV spectroscopy using synchrotron radiation as well as by cathodoluminescence studies. The VUV emission observed for the first time has been attributed to the luminescence of singlet STEs. The relatively small Stokes shift for luminescence of singlet STEs indicates that such STEs in $\mathrm{MgF}_{2}$ have nearly on-center lattice configurations.

\section{Acknowledgments}

This work was supported by DFG Grant 436 RUS 113/437/03, the Estonian Science Foundation (Grant 7274) and the European Community Research Infrastructure Action within the FP6 Program through the Contract RII3-CT-2004-506008 (IA-SFS). The authors would like to thank I A Kamenskikh, A $\mathrm{N}$ Vasil'ev and $\mathrm{G}$ Zimmerer for valuable discussions. A Kotlov is acknowledged for his assistance in measurements with VUV synchrotron radiation.

\section{References}

[1] Song K S and Williams R T 1996 Self-Trapped Excitons (Berlin: Springer)

[2] Kan'no K, Tanaka K and Yayashi T 1990 Rev. Solid State Sci. 4 383

[3] Williams R T, Kabler M N, Hayes W and Stott J P 1976 Phys. Rev. B 14725

[4] Ershov N N, Zakharov N G and Rodnyi P A 1982 Opt. Spectrosc. 5389

[5] Aleksandrov Yu M, Makhov V N, Rodnyi P A, Syrejshchikova T I and Yakimenko M N 1986 Sov. Phys.-Solid State 281598

[6] Becker J, Kirm M, Kolobanov V N, Makhov V N, Mikhailin V V, Vasil'ev A N and Zimmerer G 1998 Proc. 3rd Int. Conf. on Excitonic Processes in Condensed Matter (Boston, USA, Nov. 1998) p415

[7] Call P J, Hayes W and Kabler M N 1975 J. Phys. C: Solid State Phys. 8 L60

[8] Williams R T, Marquardt C L, Williams J W and Kabler N M 1977 Phys. Rev. B 155003

[9] Zimmerer G 2007 Radiat. Meas. 42859

[10] Kirm M, Lushchik A, Lushchik Ch, Vielhauer S and Zimmerer G 2003 J. Lumin. 102/103 307

[11] Feldbach E, Kotlov A, Kudryavtseva I, Liblik P, Lushchik A, Maaroos A, Martinson I, Nagirnyi V and Vasil'chenko E 2006 Nucl. Instrum. Methods B 250159

[12] Babin V, Feldbach E, Kirm M, Makhov V N and Vielhauer S 2008 IEEE Trans. Nucl. Sci. 551437 
[13] Tomas J, Stephan G, Lemonnier J C, Nisar M and Robin S 1973 Phys. Status Solidi b 56163

[14] Ueda Y 1976 J. Phys. Soc. Japan 411255

[15] Chen L F, Zhou L and Song K S 1997 J. Phys.: Condens. Matter 96633
[16] Ikezawa M and Kojima T 1969 J. Phys. Soc. Japan 271551

[17] Matsumoto T, Shirai M and Kan'no K 1995 J. Phys. Soc. Japan 64987

[18] Norman C D and Halliburton L E 1977 Phys. Rev. B 155883 